\title{
Actinide targets for the synthesis of super-heavy elements
}

\author{
J.B. Roberto, C.W. Alexander, R.A. Boll, J. D. Burns, J. G. Ezold, \\ L.K. Felker, S.L. Hogle, and K.P. Rykaczewski \\ Oak Ridge National Laboratory, 1 Bethel Valley Road, Oak Ridge, Tennessee 37831, USA
}

\begin{abstract}
Since 2000, six new super-heavy elements with atomic numbers 113 through 118 have been synthesized in hot fusion reactions of ${ }^{48} \mathrm{Ca}$ beams on actinide targets. These target materials, including ${ }^{242} \mathrm{Pu},{ }^{244} \mathrm{Pu},{ }^{243} \mathrm{Am},{ }^{245} \mathrm{Cm}$, ${ }^{248} \mathrm{Cm},{ }^{249} \mathrm{Cf}$, and ${ }^{249} \mathrm{Bk}$, are available in very limited quantities and require specialized production and processing facilities resident in only a few research centers worldwide. This report describes the production and chemical processing of heavy actinide materials for super-heavy element research, current availabilities of these materials, and related target fabrication techniques. The impact of actinide materials in super-heavy element discovery is reviewed, and strategies for enhancing the production of rare actinides including ${ }^{249} \mathrm{Bk},{ }^{251} \mathrm{Cf}$, and ${ }^{254} \mathrm{Es}$ are described.
\end{abstract}

Keywords: Actinide; actinide target; super-heavy element; berkelium; californium; einsteinium

This manuscript has been authored by UT-Battelle, LLC under Contract No. DE-AC05-00OR22725 with the U.S. Department of Energy. The United States Government retains and the publisher, by accepting the article for publication, acknowledges that the United States Government retains a non-exclusive, paid-up, irrevocable, worldwide license to publish or reproduce the published form of this manuscript, or allow others to do so, for United States Government purposes. The Department of Energy will provide public access to these results of federally sponsored research in accordance with the DOE Public Access Plan (http://energy.gov/downloads/doe-public-access-plan). 


\section{Introduction}

Actinides are radioactive elements ranging from atomic number 89 through 103 . First identified as a new row in the Periodic Table by Seaborg in the 1940s [1,2], most actinides are highly reactive metals with a wide range of valence states and physical properties. Thorium and uranium are the only actinides that occur in substantial quantities in nature. Actinium and protactinium were discovered around 1900 as decay products in uranium ores [3].

All actinides heavier than uranium were discovered using other actinides as targets. Neptunium (1940), plutonium (1941), americium (1944), curium (1944), berkelium (1949), and californium (1950) were first produced at the University of California, Berkeley using accelerator-based nuclear reactions with neutrons, deuterons, and alpha particles on actinide targets [4]. Einsteinium and fermium were first isolated at Berkeley in 1952 from the products of neutron irradiation of uranium in a nuclear explosion [5]. Mendelevium (1955), nobelium (1965), and lawrencium (1961-1965) were first produced in accelerators at Berkeley [6], the Joint Institute for Nuclear Research, Dubna [7,8], and Berkeley [9] and Dubna [10], respectively, all using actinide targets. Synthetic production in nuclear reactors is required to accumulate more than trace quantities of actinides heavier than uranium, with only neptunium, plutonium, americium, curium, berkelium, and californium available in the quantities $(\sim 10 \mathrm{mg})$ needed for targets for super-heavy element synthesis.

Super-heavy elements are elements with atomic numbers of 104 or greater. Nine of the 15 superheavy elements synthesized to date have been produced using actinide targets. The first super-heavy element, rutherfordium $(Z=104)$, was originally observed [11] at Dubna in 1964 using accelerator irradiation of a plutonium target with neon ions, with further work [12,13] at Dubna and Berkeley leading to formal acceptance as a new element. Dubnium $(\mathrm{Z}=105)$ was produced $[14,15]$ contemporaneously at Berkeley and Dubna in 1970, from nitrogen on californium and neon on americium, respectively. Berkeley used oxygen beams on californium to produce seaborgium $(Z=106)$ [16] in 1974 with contemporaneous work at Dubna [17]. From 1981-1996, super-heavy elements Z=107-112 were first produced at GSI, Darmstadt using non-actinide lead and bismuth targets in cold fusion reactions [18-23].

Since 2000, six new elements with atomic numbers $Z=113-118$ have been produced [24-28] using the "hot fusion" technique [29], where neutron-rich actinide targets are bombarded by neutron-rich ${ }^{48} \mathrm{Ca}$ ions, as summarized in Table 1. These elements were first produced at Dubna, with later hot fusion experiments at Berkeley confirming element 114 [30] and GSI, Darmstadt confirming elements 114 [31], 115 [32], 116 [33] and 117 [34]. Successfully pioneered and developed [29] at Dubna, hot fusion takes advantage of the increasing fission barrier height and reduced neutron separation energy as the predicted

Table 1. Hot fusion reactions using actinide $+{ }^{48} \mathrm{Ca}$ reactions have been used to produce six new super-heavy elements since 2000 . The results were originally obtained at Dubna with later confirmations at GSI and LBNL (114), and GSI (115, 116, 117). Shown is the number of nuclei directly produced as well the total production including nuclei observed in decay chains.

\begin{tabular}{|c|c|c|c|c|c|}
\hline Element (Z) & Year & Target & Projectile & $\begin{array}{l}\begin{array}{l}\text { Nuclei produced } \\
\text { (directly/total) }\end{array} \\
\end{array}$ & Reference \\
\hline (113) & 2004 & ${ }^{243} \mathrm{Am}$ & ${ }^{48} \mathrm{Ca}$ & $2 / 89$ & {$[25,32]$} \\
\hline Flerovium (114) & 2000 & ${ }^{244} \mathrm{Pu}$ & ${ }^{48} \mathrm{Ca}$ & $64 / 99$ & {$[24,30,31]$} \\
\hline (115) & 2004 & ${ }^{243} \mathrm{Am}$ & ${ }^{48} \mathrm{Ca}$ & $63 / 87$ & {$[25,32]$} \\
\hline Livermorium (116) & 2005 & ${ }^{245,248} \mathrm{Cm}$ & ${ }^{48} \mathrm{Ca}$ & $31 / 35$ & {$[26,33]$} \\
\hline (117) & 2010 & ${ }^{249} \mathrm{Bk}$ & ${ }^{48} \mathrm{Ca}$ & 22 & {$[28,34,46]$} \\
\hline (118) & 2006 & ${ }^{249} \mathrm{Cf}$ & ${ }^{48} \mathrm{Ca}$ & 4 & [27] \\
\hline
\end{tabular}


closed shell at $\mathrm{N}=184$ is approached. This allows the compound nucleus to stabilize by shedding 2-4 neutrons, resulting in increased survivability in comparison to compound nuclei from cold fusion reactions for $\mathrm{Z}=113$ or greater. The use of actinide ${ }^{48} \mathrm{Ca}$ reactions has increased the production rate for super-heavy elements with $Z=113$ or greater by one or more orders of magnitude, reducing required accelerator times from years to months and enabling the discovery of elements up to $Z=118$ and possibly higher. Element 113 has also been produced using a non-actinide target, in a multi-year experiment using a zinc beam on a bismuth target at RIKEN, Wako [35]. Additional information on the observation and identification of isotopes with $\mathrm{Z} \geq 100$ can be found in [36].

\section{Production and chemical processing}

\subsection{Production and availability of actinides}

Actinide targets for super-heavy element synthesis using ${ }^{48} \mathrm{Ca}$ beams have included ${ }^{238} \mathrm{U},{ }^{237} \mathrm{~Np}$, ${ }^{242,244} \mathrm{Pu},{ }^{243} \mathrm{Am},{ }^{245,248} \mathrm{Cm},{ }^{249} \mathrm{Bk}$, and ${ }^{249} \mathrm{Cf}$, with all target materials heavier than uranium produced in nuclear reactors. Many of these actinide materials have been obtained from Oak Ridge National Laboratory (ORNL) and from the Research Institute for Advanced Reactors (RIAR) at Dmitrovgrad. At ORNL, the combination of the High Flux Isotope Reactor (HFIR) and the Radiochemical Engineering Development Center (REDC) provides a unique resource for the production and chemical processing of actinides. ORNL is also the repository for actinide isotopes for the Isotope Development and Production for Research and Applications Program of the U.S. Department of Energy (DOE) Office of Nuclear Physics. Current inventories of selected actinide materials at ORNL are summarized in Table 2.

\subsection{Reactor production of heavy actinides}

Heavy actinides are produced in HFIR by intense neutron irradiation of mixed americium and curium targets. The primary production isotope, ${ }^{252} \mathrm{Cf}$, is produced for industrial, medical, and research applications on a regular 2-year schedule. Up to $200 \mathrm{mg}$ of ${ }^{252} \mathrm{Cf}$ is produced in each campaign. This process also produces $\sim 20 \mathrm{mg}$ of ${ }^{249} \mathrm{Bk}$, micrograms of ${ }^{254} \mathrm{Es}$, and picograms of ${ }^{257} \mathrm{Fm}$. The $\mathrm{Am} / \mathrm{Cm}$ source material was produced nearly 40 years ago via multi-stage irradiation of plutonium in the now

Table 2. Selected actinide inventories at ORNL. Actinide isotopes from ORNL have been involved in the discovery of elements $104-106[13,14,16]$ and 113-118 [37].

\begin{tabular}{|c|c|c|c|}
\hline Isotope & $\begin{array}{l}\text { Approximate } \\
\text { amount (mg) }\end{array}$ & Isotopic \% & Notes \\
\hline${ }^{237} \mathbf{N p}$ & 1000 & $>99 \%$ & \\
\hline${ }^{242} \mathbf{P u}$ & 5500 & $>99 \%$ & \\
\hline${ }^{241} \mathrm{Am}$ & 3500 & $>99 \%$ & \\
\hline${ }^{243} \mathrm{Am}$ & 1000 & $>99 \%$ & \\
\hline${ }^{244} \mathrm{Cm}$ & 1000 & $>90 \%$ & \\
\hline${ }^{248} \mathrm{Cm}$ & 2500 & $80-95 \%$ & $80 \mathrm{mg}$ at $95 \%$ in stock, remaining requires recovery processing \\
\hline${ }^{249} \mathbf{B k}$ & - & $>99 \%$ & $\begin{array}{l}\text { Requires HFIR production and REDC chemical processing } \\
\text { ( 20 mg per Cf campaign) }\end{array}$ \\
\hline${ }^{249} \mathbf{C f}$ & 170 & $>99 \%$ & \\
\hline${ }^{251} \mathrm{Cf}$ & 150 & $\sim 35 \%$ & $\begin{array}{l}\text { Requires recovery from old }{ }^{252} \mathrm{Cf} \text { sources and chemical processing } \\
\text { (isotopic separation required for higher enrichment) }\end{array}$ \\
\hline
\end{tabular}


decommissioned K-reactor at the Savannah River Site. A portion of this material was reserved for heavy actinide production at ORNL, and through decades of recycling the ORNL material has become rich in ${ }^{246} \mathrm{Cm}$, and to a lesser extent ${ }^{248} \mathrm{Cm}$. Additional material available at ORNL for heavy actinide production contains larger weight fractions of the lighter isotopes ${ }^{244} \mathrm{Cm}$ and ${ }^{243} \mathrm{Am}$, and accordingly would require more extensive irradiation and chemical processing.

The ${ }^{252} \mathrm{Cf}$ production process begins with conversion of the $\mathrm{Am} / \mathrm{Cm}$ source material to oxide microspheres that are blended with aluminum powder and pressed to form cermet pellets. These pellets are loaded into long, thin, finned aluminum tubes (targets) that are closure welded and then hydrostatically compressed to provide good heat transfer between the pellets and target tubes. A final aluminum jacket is wrapped around the target tube to channel the coolant flow around the target during irradiation (Fig. 1). The amount of actinide material in each target is limited both in mass, to reduce heat

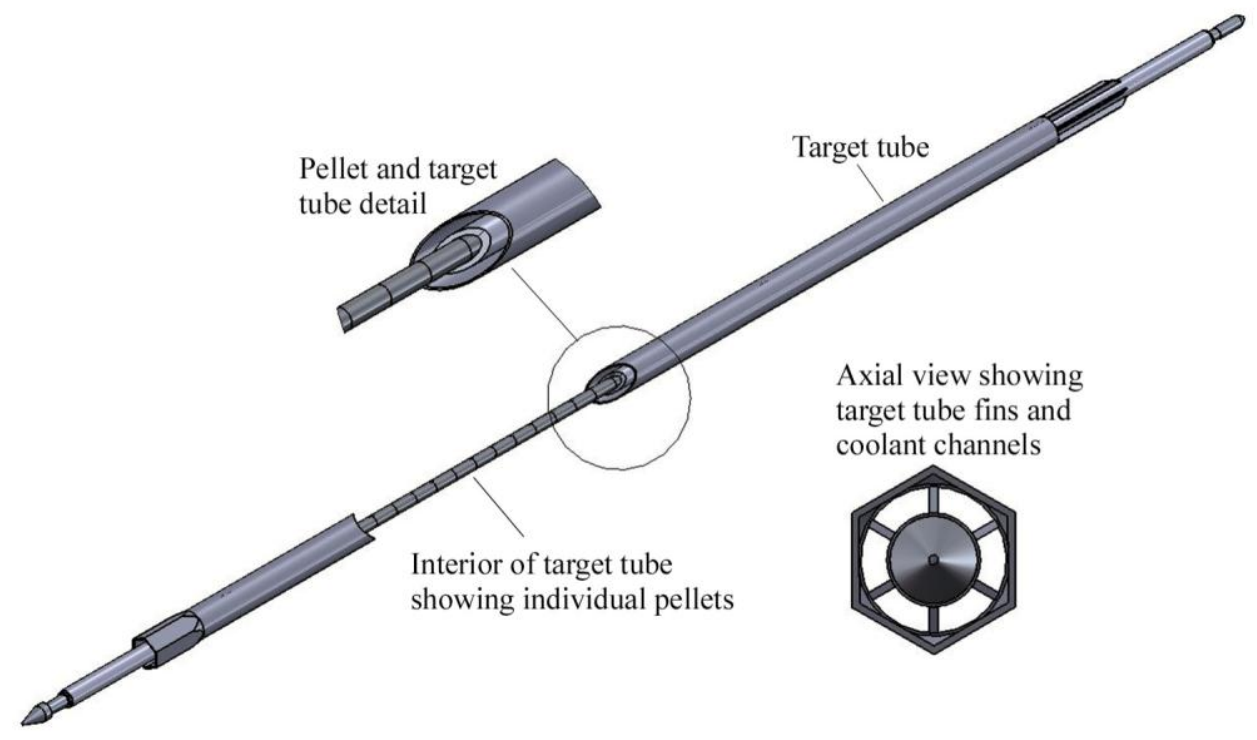

Fig. 1. Californium production target

generation, and volume, to maintain heat transfer through the aluminum matrix and allow for containment of fission gasses. Each target generally contains 5-8 grams of Am/Cm.

The target rods are inserted into the flux trap of HFIR, where they are exposed to an intense thermal neutron flux of $3 \times 10^{15} \mathrm{n} / \mathrm{s}-\mathrm{cm}^{2}$. The Am/Cm is transmuted into heavier isotopes by a series of neutron captures and beta decays (Fig. 2), with losses due to fission and decay. Because of the many neutron captures required to produce ${ }^{252} \mathrm{Cf}$, each with its own energy-dependent cross section, target consumption and actinide production are sensitive to the neutron energy spectrum as well as the neutron flux. Transmutation rates vary as isotopes accumulate and/or deplete, and modeling and simulation is required to optimize transmutation over the projected duration of irradiation and select an irradiation plan. This plan may focus on maximizing ${ }^{252} \mathrm{Cf}$ production, minimizing target material fission losses, increasing the isotopic fraction of heavier curium isotopes, or some combination of these and other factors.

A typical ${ }^{252} \mathrm{Cf}$ irradiation plan involves placement of targets in positions of maximum total neutron flux for three to five 24 -day reactor cycles. Under these conditions, ${ }^{252} \mathrm{Cf}$ precursors ${ }^{249} \mathrm{Bk},{ }^{250} \mathrm{Cf}$, and ${ }^{251} \mathrm{Cf}$ reach their maximum accumulation rate after approximately one cycle, and accumulation of ${ }^{252} \mathrm{Cf}$ 


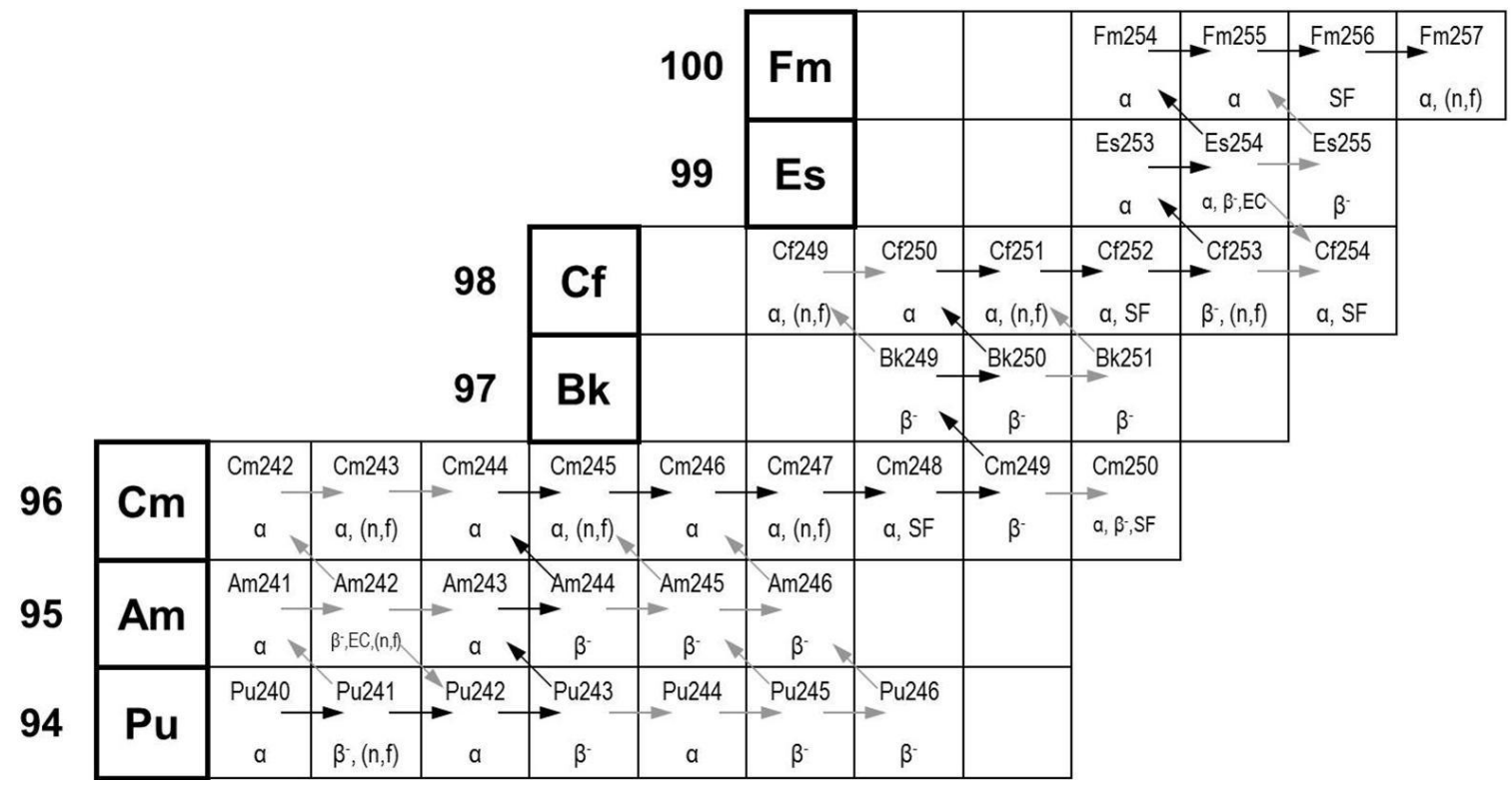

Fig 2. Reactor production of transcurium actinides from multiple neutron captures and beta decays at HFIR Dark arrows show the primary reaction channel, while light arrows show lesser channels.

reaches its maximum rate during the second cycle. Further irradiation results in additional accumulation of ${ }^{252} \mathrm{Cf}$, but at an ever-decreasing rate. The rate of feedstock consumption via fission does not decrease with time, and hence production efficiency, in terms of heavy actinides produced per unit of target consumption, is highest in the first two cycles. Fig. 3 shows the accumulation of ${ }^{252} \mathrm{Cf}$ during a typical production campaign, as well as the yield of ${ }^{252} \mathrm{Cf}$ per fission in the target.

For production of ${ }^{249} \mathrm{Bk}$, a typical irradiation plan would place targets in positions of maximum epithermal-to-thermal neutron flux ratio for a single cycle. Under this irradiation scheme ${ }^{249} \mathrm{Bk}$ reaches its maximum accumulation rate early in the cycle, with a total ${ }^{249} \mathrm{Bk}$ accumulation over $90 \%$ of the

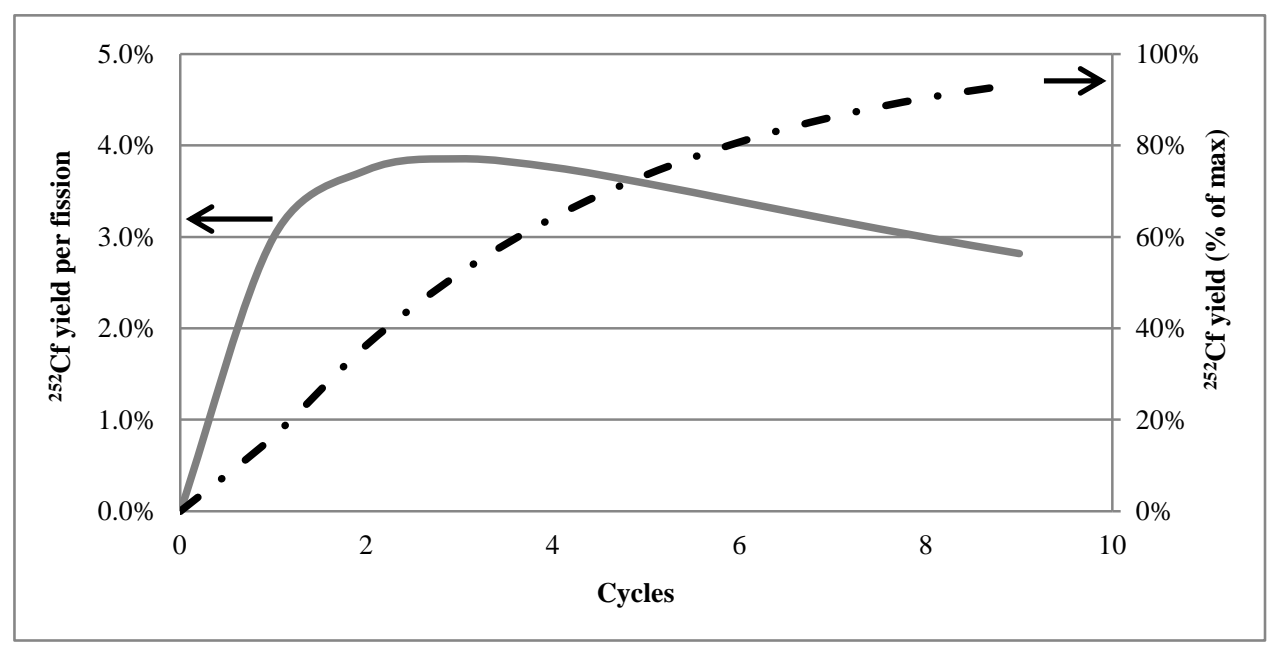

Fig. $3 .{ }^{252} \mathrm{Cf}$ yield as a function of the number of reactor cycles in HFIR The yield per fission reaches its maximum during the second cycle (solid line), with total ${ }^{252} \mathrm{Cf}$ accumulation growing at a decreasing rate thereafter (dashed line). 
maximum possible by the end of the cycle. Irradiation for a second cycle results in minor increases in ${ }^{249} \mathrm{Bk}$ accumulation, additional irradiation results in depletion of the accumulated ${ }^{249} \mathrm{Bk}$ as precursor $\mathrm{Cm}$ isotopes are depleted.

After irradiation, ${ }^{252} \mathrm{Cf}$ production targets are typically allowed to cool in the HFIR storage pool for three months while fission and activation products decay. Decay time is largely constrained by the ${ }^{131} \mathrm{I}$ activity, a highly dispersible and radiotoxic fission product. Required decay times can be reduced by minimizing the total fission in the targets. Because of the high fission cross sections of ${ }^{245} \mathrm{Cm}$ and ${ }^{247} \mathrm{Cm}$, target materials with a higher weight percent of ${ }^{244} \mathrm{Cm}$ result in higher fission product yields, and hence require additional cooling. During production of ${ }^{249} \mathrm{Bk}$ the use of $\mathrm{Cm}$ blends rich in ${ }^{248} \mathrm{Cm}$ allows not only faster production of ${ }^{249} \mathrm{Bk}$, but also faster post-irradiation processing, due to minimizing production of ${ }^{131} \mathrm{I}$ and other fission products.

\subsection{Chemical processing of irradiated targets}

The chemical processing of neutron-irradiated targets to recover $\mathrm{Cf}$ and $\mathrm{Bk}$ is illustrated in Fig. 4. Once the Am/Cm targets have been irradiated in HFIR and allowed to cool, they are transferred to REDC for separation and purification of transcurium actinides. The aluminum components are dissolved in a sodium hydroxide $(\mathrm{NaOH})$ and sodium nitrate $\left(\mathrm{NaNO}_{3}\right)$ mixture [38,39]. The targets are placed in 2.1 $\mathrm{MNaNO}_{3}$ and heated to $92{ }^{\circ} \mathrm{C}$, at which point $10 \mathrm{MNaOH}$ is slowly added, so that the final concentration is $\sim 1.5 \mathrm{M} \mathrm{NaNO}_{3}$ and $3.0 \mathrm{M} \mathrm{NaOH}$. The temperature of the solution is then increased to $104{ }^{\circ} \mathrm{C}$ and maintained for 30 minutes. After cooling, the mixture contains dissolved aluminum as $\mathrm{Al}(\mathrm{OH})_{4}$ and precipitated metal hydroxides which contain the actinides and lanthanides. The solids are filtered and washed several times with $1 \mathrm{M} \mathrm{NaOH}$, followed by several water washes. They are then dissolved in $8.0 \mathrm{M}$ nitric acid $\left(\mathrm{HNO}_{3}\right)$ at $103{ }^{\circ} \mathrm{C}$, using an air sparge to facilitate mixing and accelerate

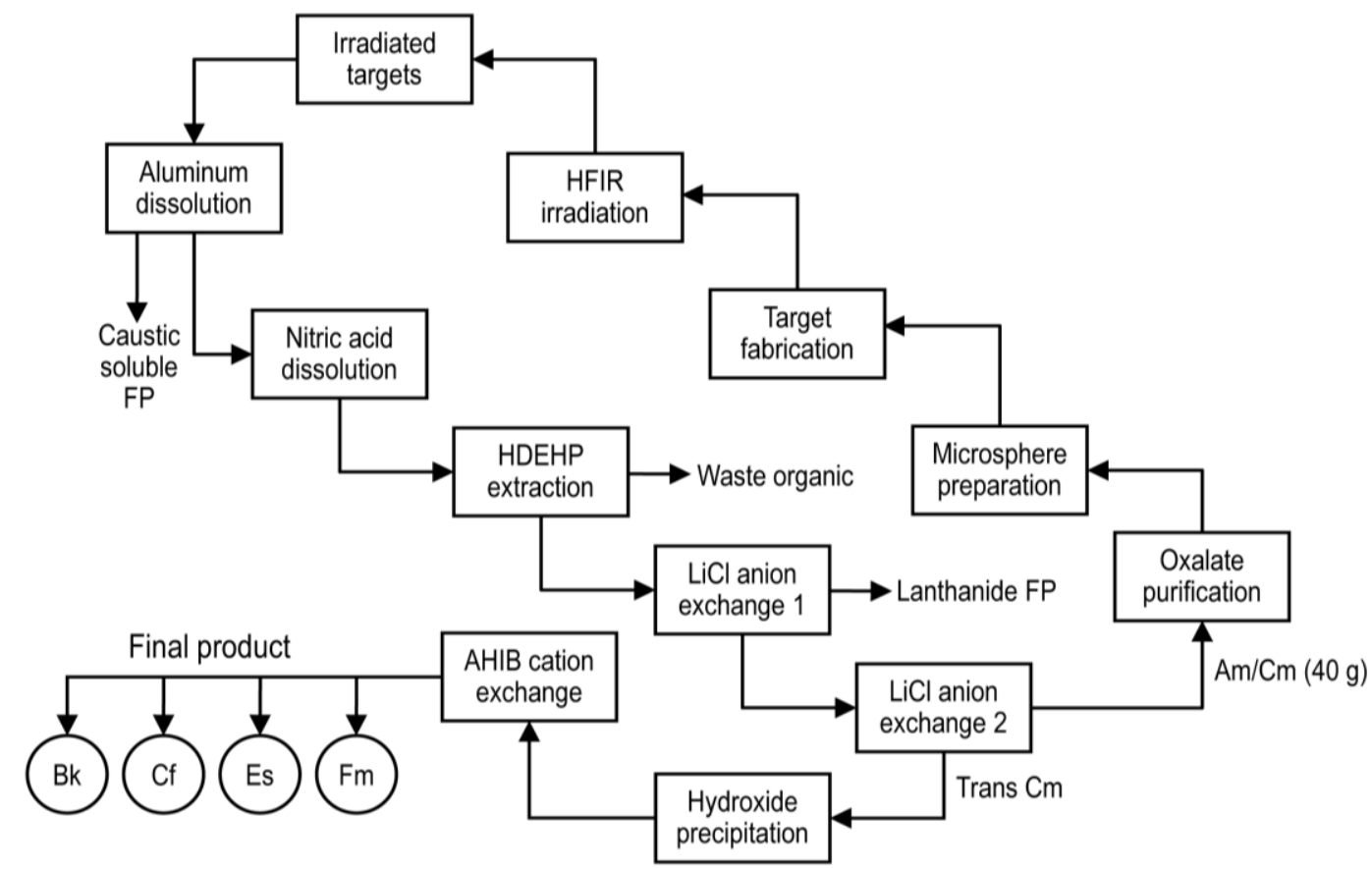

Fig. 4. Process flow for production and chemical separation of transcurium actinides. Typical yields at HFIR/REDC are up to $200 \mathrm{mg}$ of ${ }^{252} \mathrm{Cf}$, 20mg of ${ }^{249} \mathrm{Bk}$, micrograms of ${ }^{254} \mathrm{Es}$, and picograms of ${ }^{257} \mathrm{Fm}$. 
dissolution. After dissolution, all of the desired actinide materials are in solution, and any remaining undissolved solids are filtered and discarded.

The actinide solution still contains many other metals that need to be separated and either recycled or discarded. The next step is a batch solvent extraction procedure utilizing di-(2-ethylhexyl) phosphoric acid (HDEHP) [40]. The actinide solution is first brought to near dryness to drive off excess $\mathrm{HNO}_{3}$, and a small amount of concentrated hydrochloric acid $(\mathrm{HCl})$ is added. The solution is then heated to $120{ }^{\circ} \mathrm{C}$ to ensure dissolution of any hydrolyzed materials produced by contact with $\mathrm{HNO}_{3}$. After cooling, the solution is diluted with water, and the hydrogen ion concentration is adjusted to $0.1-0.4 \mathrm{~N}$. An oxidant, $1.5 \mathrm{M}$ sodium hypochlorite $(\mathrm{NaOCl})$, is added to make the solution $0.1 \mathrm{M} \mathrm{NaOCl}$. The solution is heated to $80{ }^{\circ} \mathrm{C}$ for approximately $20 \mathrm{~min}$ to oxidize molybdenum to $\mathrm{Mo}(\mathrm{VI})$ and ensure its extraction, avoiding hydrolysis problems later in the process. The actinides, lanthanides, and molybdenum are batch extracted with 1.0 $M$ HDEHP in normal paraffin hydrocarbons (NPH) at an aqueous to organic (A:O) phase ratio of nominally $1: 2$. As the extraction progresses, the acidity of the aqueous phase is reduced to $0.03 \mathrm{~N}$ with $\mathrm{NaOH}$ to increase actinide extraction. The pregnant organic phase is then scrubbed twice with $0.03 \mathrm{~N} \mathrm{HCl}$ at an A:O phase ratio of 1:3. After scrubbing, Adogen ${ }^{\mathrm{TM}} 364-\mathrm{HP}$, an amine compound, is added to the organic phase to prevent iron back-extraction during stripping. The actinides are stripped from the organic phase with $6.0 \mathrm{M} \mathrm{HCl}-0.5 \mathrm{M} \mathrm{H}_{2} \mathrm{O}_{2}$. The hydrogen peroxide is crucial for recovery of berkelium, because it counteracts any radiolytic oxidation that the berkelium may have undergone.

Lanthanide separation. A lithium chloride ( $\mathrm{LiCl}$ )-based anion exchange procedure separates the lanthanides from the actinides [41,42]. The stripped aqueous phase from the previous step serves as the feed. It is pre-washed with an organic diluent, diethylbenzene, to remove any remaining extractant that might degrade during evaporation, and then evaporated to a small volume. Lithium chloride is added, resulting in $\sim 13 \mathrm{M} \mathrm{LiCl}$ concentration, and the solution is heated to $142.5^{\circ} \mathrm{C}$, the boiling point of $13 M \mathrm{LiCl}$. After cooling, concentrated $\mathrm{HCl}$ is added to make the feed $12 M \mathrm{LiCl}-1.0 \mathrm{M} \mathrm{HCl}$. Insoluble aluminum, sodium, and zirconium precipitates form and are filtered. The filtered solids are washed four times with $12 \mathrm{M} \mathrm{LiCl}-1.0 \mathrm{M} \mathrm{HCl}$, and the feed is readjusted to $\sim 13 \mathrm{M} \mathrm{LiCl}$ by boiling at $142.5^{\circ} \mathrm{C}$. After cooling, concentrated $\mathrm{HCl}$ is again added to make the solution $1 \mathrm{M} \mathrm{HCl}$, but this time the solution is heated to $120^{\circ} \mathrm{C}$. At this temperature, $\mathrm{HCl}$ is evaporated and the solution adjusts itself to $\sim 12 \mathrm{M} \mathrm{LiCl}-$ $0.1 \mathrm{M} \mathrm{HCl}$, as evidenced by $\mathrm{AlCl}_{3}$ dropping out of solution. The feed is loaded onto a column packed with cation ion exchange resin, Dowex 1X10, which has been preconditioned with 4 bed volumes of $12 M \mathrm{LiCl}-0.1 M \mathrm{HCl}$.

After loading is completed, the lanthanides are eluted in the first fraction with 4 bed volumes of $10 \mathrm{M} \mathrm{LiCl}$. Next, the bulk of americium and curium, $90-95 \%$, is eluted in the second fraction with 3 bed volumes of $9 \mathrm{M} \mathrm{LiCl}$. Then the heavier actinides (Bk, Cf, Es, and $\mathrm{Fm}$ ) with the remaining americium and curium are eluted in the third fraction using 2 bed volumes of $8 M \mathrm{HCl}$. Finally, the column is stripped of any sorbed impurities, such as zirconium or plutonium, with 3 bed volumes of $0.8 \mathrm{M} \mathrm{HCl}$. During the column run, the elution of heavier actinides is tracked by a neutron profile of the column and by observation of the alpha activity of column eluent. Since Bk is a pure beta emitter, its location is estimated to be after the $\mathrm{Cm}$ and before the Cf. The third fraction is processed through a second $\mathrm{LiCl}$ column to enhance the separation of the $\mathrm{Bk}$ and $\mathrm{Cf}$ from the 5-10\% of Am and Cm collected with the heavier actinides. In this second $\mathrm{LiCl}$ run, the transcurium products are eluted in $1 \mathrm{M} \mathrm{HCl}$, rather than $8 \mathrm{M} \mathrm{HCl}$. The Am and $\mathrm{Cm}$ from both columns are stored to await conversion into targets for future irradiations, while the transcurium products are further purified.

Actinide separation. The individual actinides are separated from each other using $\alpha$ hydroxyisobutyric acid $(\alpha-\mathrm{HIB})$ and cation exchange $[43,44]$. The transcurium product is converted from the chloride to nitrate form by precipitating the actinides using $\mathrm{LiOH}$ and then filtering the solids. The transcurium precipitate is dissolved in a small volume of $8 \mathrm{MHNO}_{3}$ and diluted with water to $0.3 \mathrm{MHNO}_{3}$. This solution serves as the feed for the $\alpha$-HIB-cation exchange column separation. The column separation is set up with two columns in series: a small load column at the front, followed by a 
larger elution column (5.8 times the size of the load column). This design minimizes the amount of waste generated by radiolytic resin degradation, so that only the resin in the load column needs to be changed after every run. Both columns are packed with Dowex 50X8 and conditioned with $0.3 M \mathrm{NH}_{4} \mathrm{NO}_{3}$ to convert the resin to the ammonium form. Optimal separation occurs at elevated temperatures $\left(\sim 70{ }^{\circ} \mathrm{C}\right)$, so in this configuration only the elution column needs to be heated. The feed solution is passed through the load column at a rate of $10 \pm 1.5$ bed volumes per hour. After loading, the column is washed with $\mathrm{H}_{2} \mathrm{O}$, then with 1.75 bed volumes of $0.3 \mathrm{MNH}_{4} \mathrm{NO}_{3}$, and finally with $\mathrm{H}_{2} \mathrm{O}$ again to ensure that the resin is still in the ammonium form. After the actinides are loaded, the outlet of the load column is connected to the inlet of the elution column.

To begin eluting the actinides, $0.25 M \alpha$-HIB-pH 4.2 is pumped through the columns at 5.8 bed volumes per hour. The $\mathrm{Cf}$ normally elutes in $10 \pm 1.5$ bed volumes. After the $\mathrm{Cf}$ elutes, the elution solution is changed to $0.25 M \alpha$-HIB-pH 4.6, and the Bk normally elutes in 4 bed volumes. Any tailings of $\mathrm{Am}$ and $\mathrm{Cm}$ not separated in the $\mathrm{LiCl}$ anion exchange are stripped from the column using 2.5 bed volumes of $0.5 \mathrm{M} \alpha$-HIB-pH 4.8, and the column is reconstituted with a water wash. For storage, the Cf fraction is loaded onto small amounts of cation exchange resin in $1 \mathrm{~mL}$ platinum columns. The loaded columns are dried for 24 hours and then fired to $700{ }^{\circ} \mathrm{C}$. The fired packages are sealed with Swagelok ${ }^{\mathrm{TM}}$ fittings on either end and held for future product requests.

Berkelium recovery. Additional steps are required to recover a purified berkelium product free from any californium contamination [39]. The Bk fraction from the previous $\alpha H I B$ actinide separation step is concentrated by passing through a cation ion exchange column, washed with 8 bed volumes of $\mathrm{H}_{2} \mathrm{O}$ to remove all of the $\alpha$-HIB reagent, and then stripped with 20 bed volumes of $8 M \mathrm{HNO}_{3}$. The Bk is oxidized from $\mathrm{Bk}(\mathrm{III})$ to $\mathrm{Bk}(\mathrm{IV})$ by adding $2 \mathrm{M}$ sodium bromate $\left(\mathrm{NaBrO}_{3}\right)$ to make the solution $0.3 \mathrm{M} \mathrm{NaBrO}_{3}$. The $\mathrm{Bk}(\mathrm{IV})$ is then extracted into $0.5 \mathrm{M}$ HDEHP in dodecane with an A:O phase ratio of $1: 1$, and the solution mixture is agitated by air sparging for $30 \mathrm{~min}$. The phases are allowed to separate, the aqueous phase is removed, and the organic phase is scrubbed with $8 M \mathrm{HNO}_{3}-0.3 M \mathrm{NaBrO}_{3}$ in an A:O phase ratio of 1:1. Again, the two phases are separated and the $\mathrm{Bk}(\mathrm{IV})$ is stripped from the organic phase using two strips of $8 M_{\mathrm{HNO}_{3}-1}^{-1} \mathrm{M} \mathrm{H}_{2} \mathrm{O}_{2}$ in a phase ratio of roughly $1: 1.75$. The $\mathrm{H}_{2} \mathrm{O}_{2}$ reduces $\mathrm{Bk}(\mathrm{IV})$ to $\mathrm{Bk}(\mathrm{III})$, ensuring that it is stripped back into the aqueous phase. At this point, the decontamination factor for ${ }^{252} \mathrm{Cf}$ and other radioactive impurities in the Bk product is $\sim 10^{5}$, and the solution can be safely transferred to a glove box for further processing.

Once in the glove box, the Bk solution is washed three times with trichloroethylene (TCE) to remove any HDEHP/dodecane remaining from the solvent extraction procedure. The Bk solution is then evaporated, dissolved in dilute $\mathrm{HCl}$, and processed through a small $\alpha$-HIB-cation column using the procedure described above. This separates the $\mathrm{Bk}$ from any remaining $\mathrm{Cf}$. The purified $\mathrm{Bk}$ fraction is acidified to $0.3 \mathrm{M} \mathrm{HCl}$ and loaded onto a $3 \mathrm{~mL}$ cation exchange resin column, AG50X4, with an internal diameter of $\sim 1 \mathrm{~cm}$. The column is washed with $0.1 \mathrm{M} \mathrm{HCl}$, then $2 \mathrm{M} \mathrm{HCl}$. This removes mono- and divalent metals, iron, and $\alpha$-HIB reagent from the Bk. The ultrapure $\mathrm{Bk}$ is then stripped with $8 \mathrm{M} \mathrm{HCl}$ from the column, evaporated, and analyzed. The separated Bk includes $<10^{-9} \mathrm{~g}$ of ${ }^{252} \mathrm{Cf}$, with $\sim 10^{7}$ or greater decontamination factors for all radionuclides, and is ready for shipment.

\section{Actinide materials for super-heavy element research}

\subsection{Plutonium, americium, and curium}

Transuranic actinides, including $\mathrm{Pu}, \mathrm{Am}$, and $\mathrm{Cm}$, have been used for discovery research on elements 104 and 105 using actinide $+{ }^{22} \mathrm{Ne}$ reactions $[11,15]$ and elements 114,115 , and 116 using actinide $+{ }^{48} \mathrm{Ca}$. [24-26]. These actinide isotopes generally have long half-lives (18.1y for ${ }^{244} \mathrm{Cm}$, hundreds of years and greater for the others) and can be recovered and recycled after completion of an experiment. Inventories 
at ORNL (Table 2) are substantially greater than the tens of milligrams required for typical super-heavy element experiments.

\subsection{Berkelium}

The synthesis of element 117 was first reported [28] in 2010 by a Russia-U.S. team using the ${ }^{249} \mathrm{Bk}+{ }^{48} \mathrm{Ca}$ reaction at the Dubna Gas-Filled Recoil Separator. This experiment utilized $15 \mathrm{mg}$ of ${ }^{249} \mathrm{Bk}$ produced and separated at HFIR/REDC at ORNL. Subsequent experiments at Dubna and GSI confirmed these results, again using ${ }^{249} \mathrm{Bk}$ produced at ORNL [45,46,34]. ${ }^{249} \mathrm{Bk}$ targets from ORNL have also been used for single-atom chemistry studies [47] of element 117 decay chain products at Dubna, and for a search [48] for element 119 using a ${ }^{50} \mathrm{Ti}$ beam at GSI.

${ }^{249} \mathrm{Bk}$ decays by beta emission to ${ }^{249} \mathrm{Cf}$ with a half-life of 327 days [49]. As a result, ${ }^{249} \mathrm{Bk}$ must be freshly produced for each experiment, and significant ingrowth of ${ }^{249} \mathrm{Cf}$ can occur, $\sim 30 \%$ after six months. The effect of this ingrowth was directly observed in 2012 with the production of an element 118 decay chain during an element 117 experiment [45]. The ${ }^{249} \mathrm{Bk}$ target had partially decayed to ${ }^{249} \mathrm{Cf}$, allowing the ${ }^{249} \mathrm{Cf}+{ }^{48} \mathrm{Ca}$ reaction to occur.

${ }^{249} \mathrm{Bk}$ is normally produced during ${ }^{252} \mathrm{Cf}$ campaigns at HFIR, allowing shared use of feedstock, irradiation targets, and processing steps. These campaigns typically require 3-5 reactor cycles ( $\sim 24$ days each), while less than one cycle is required to reach secular equilibrium for ${ }^{249} \mathrm{Bk}$ production. As a result, ${ }^{249} \mathrm{Bk}$ produced during multi-cycle ${ }^{252} \mathrm{Cf}$ campaigns is no greater than could be achieved in 1 -cycle irradiations. The yield of ${ }^{249} \mathrm{Bk}$ can be increased by increasing the initial fraction of ${ }^{248} \mathrm{Cm}$ in the feedstock and by maximizing the epithermal to thermal neutron flux, as described below and in section 2.2. Increasing the ${ }^{248} \mathrm{Cm}$ fraction also minimizes fission products, which reduces post irradiation processing times.

The $(\mathrm{n}, \gamma)$ neutron capture cross section and resonance integral for ${ }^{249} \mathrm{Bk}$ are $746 \mathrm{~b}$ and $1100 \mathrm{~b}$, respectively, while the cross section and resonance integral for ${ }^{248} \mathrm{Cm}$, the primary precursor to ${ }^{249} \mathrm{Bk}$, are $2.6 \mathrm{~b}$ and $270 \mathrm{~b}$ [50]. These fundamental nuclear data, along with the thermal and epithermal flux, can be combined to calculate the maximum yield of ${ }^{249} \mathrm{Bk}$, where production and destruction rates are equal.

$$
N_{C m 248} \varphi_{t h}\left(\sigma_{C m 248}+\frac{1}{r} R I_{C m 248}\right)=N_{B k 249} \varphi_{t h}\left(\sigma_{B k 249}+\frac{1}{r} R I_{B k 249}\right)+N_{B k 249} \lambda_{B k 249}
$$

In this equation $N$ is the number of atoms, $\varphi_{t h}$ is the thermal flux, $\sigma$ is the thermal cross section, $r$ is the thermal to epithermal ratio, $R I$ is the resonance integral, and $\lambda$ is the decay constant. While increasing the total neutron flux can help offset destruction of ${ }^{249} \mathrm{Bk}$ due to decay, more significant gains can be achieved by decreasing the thermal to epithermal neutron flux ratio. By reducing absorption in the thermal energy spectrum relative to absorption in the epithermal range, the rate of depletion of ${ }^{249} \mathrm{Bk}$ can be significantly reduced. However, the rate of production from ${ }^{248} \mathrm{Cm}$, with a resonance integral two orders of magnitude greater than the thermal cross section, is largely unaffected. Fig. 5 shows the absorption cross-sections for ${ }^{248} \mathrm{Cm}$ and ${ }^{249} \mathrm{Bk}$ and the approximate location $(0.5 \mathrm{eV})$ where the ratio between these cross-sections shifts significantly. Any filter used to depress the flux would need to act primarily to the left of this line.

Reduction of the neutron flux below $0.5 \mathrm{eV}$ can be achieved by the use of a filter material with a large thermal cross section and low resonance integral, such as ${ }^{113} \mathrm{Cd}$. While ${ }^{113} \mathrm{Cd}$ has been used extensively for short duration irradiations, primarily for measurement of epithermal cross sections, longer duration irradiations result in significant depletion of the Cd filter. Small scale experiments using $0.1 \mathrm{~mm}$ thick filters of ${ }^{103} \mathrm{Rh}$ in the hydraulic tube of the HFIR for up to 25 days have shown average thermal flux depressions of approximately $20 \%$ [52]. A thermal flux depression in the range of $50 \%$ for 1 cycle is considered achievable, with further research necessary to develop designs for greater flux reductions or durations. 


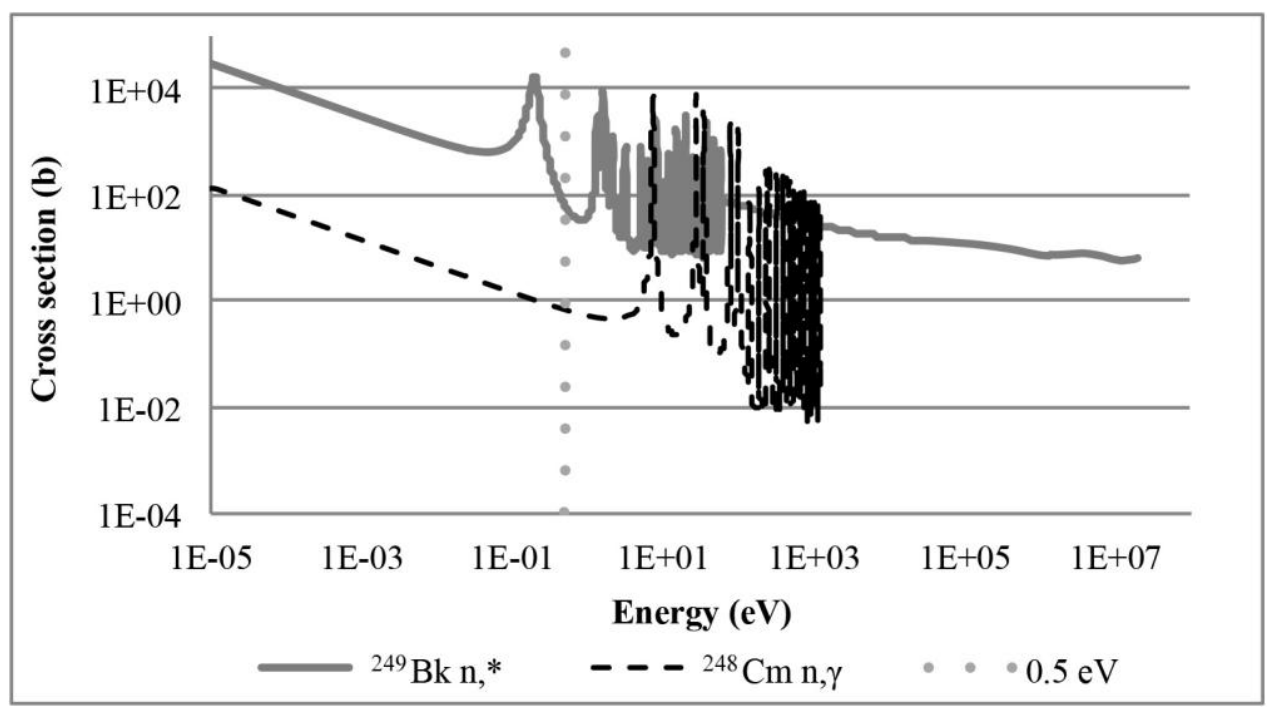

Fig. 5. Absorption cross sections [51] for ${ }^{249} \mathrm{Bk}$ and ${ }^{248} \mathrm{Cm}$ as a function of neutron energy

Fig. 6 shows the yield of ${ }^{249} \mathrm{Bk}$ as a percentage of ${ }^{248} \mathrm{Cm}$ in the target under a typical HFIR neutron flux, and under a neutron flux that has been suppressed by $50 \%$ in the thermal spectrum. In this case the yield of ${ }^{249} \mathrm{Bk}$ can be increased from $0.6 \%$ to $1 \%$ of the ${ }^{248} \mathrm{Cm}$ content in the target. This yield can be increased by up to an order of magnitude by further reduction of the thermal neutron flux. However, there are many challenges associated with the use of neutron filters that must be addressed before production using these techniques could be accomplished, including heat dissipation, possible detrimental effects on HFIR reactivity, and the flux depression potential of the materials over the duration of the irradiation as the filter is depleted.

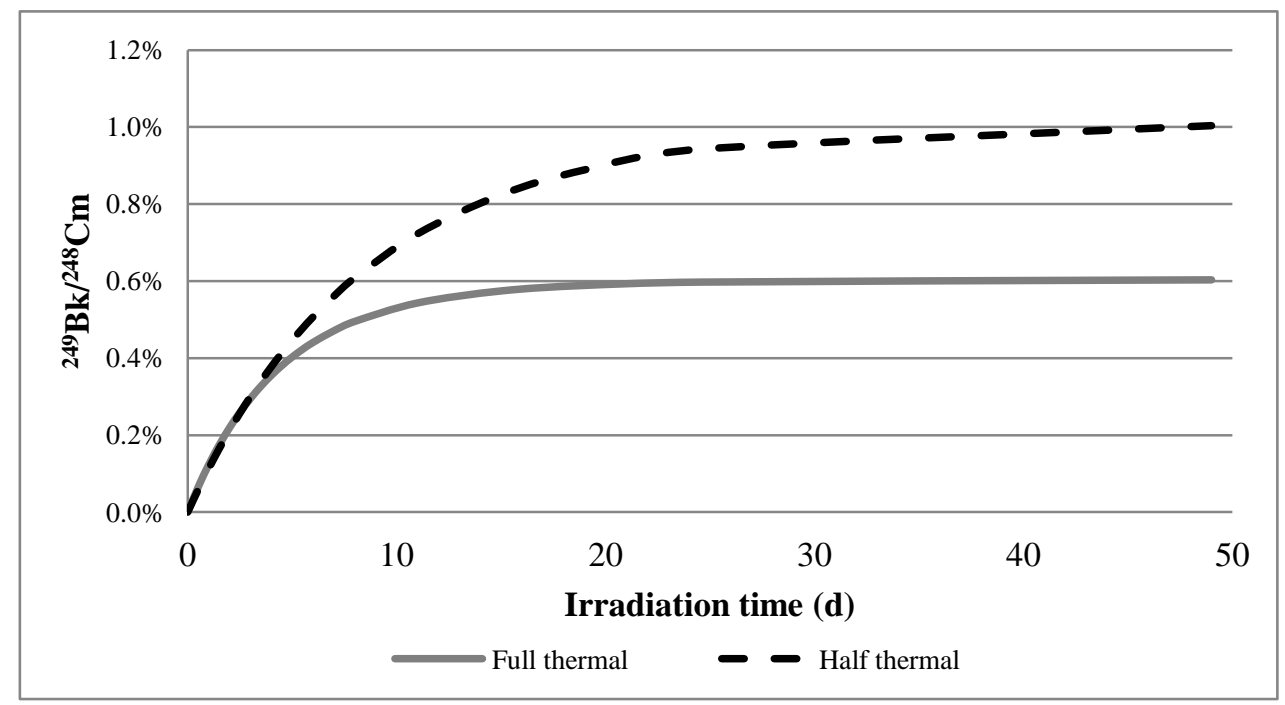

Fig. 6. ${ }^{249} \mathrm{Bk}$ yield as a percentage of ${ }^{248} \mathrm{Cm}$ content in the target for a typical and suppressed thermal neutron spectrum in HFIR 


\subsection{Californium}

Since the beginning of operations at HFIR/REDC in 1966, seventy-five campaigns have been completed to produce transcurium actinides, with ${ }^{252} \mathrm{Cf}$ being produced in the largest quantity. During irradiations for the production of ${ }^{252} \mathrm{Cf}$, the other californium isotopes, ${ }^{249} \mathrm{Cf},{ }^{250} \mathrm{Cf}$, ${ }^{251} \mathrm{Cf},{ }^{253} \mathrm{Cf}$, and ${ }^{254} \mathrm{Cf}$, are also produced. A typical distribution for californium product is shown in Table 3 with corresponding half-lives.

Table 3. Typical Cf product isotopic distribution from HFIR campaigns

\begin{tabular}{lcc}
\hline Isotope & Atomic $\%$ & Half-life (y) \\
\hline${ }^{249} \mathrm{Cf}$ & 3.41 & 351 \\
${ }^{250} \mathrm{Cf}$ & 8.70 & 13.08 \\
${ }^{251} \mathrm{Cf}$ & 2.60 & 898 \\
${ }^{252} \mathrm{Cf}$ & 85.27 & 2.645 \\
${ }^{253} \mathrm{Cf}$ & 0.004 & 0.049 \\
${ }^{254} \mathrm{Cf}$ & 0.010 & 0.166 \\
\hline
\end{tabular}

The ${ }^{253,254} \mathrm{Cf}$ isotopes decay in a relatively short time leaving ${ }^{249-252} \mathrm{Cf}$ in the californium product used for the fabrication of neutron sources in REDC. As mentioned previously, these sources are provided to industry, universities, and government laboratories for a variety of research and industrial applications. When no longer needed, the decayed sources are returned to ORNL and placed in a storage pool for potential future use. Many of these sources have been in the pool for several decades, and the ${ }^{252} \mathrm{Cf}$ has decayed to ${ }^{248} \mathrm{Cm}$, which is attractive for chemistry research and as a target for super-heavy element research.

Recently, the super-heavy element research community has expressed interest in a target of ${ }^{251} \mathrm{Cf}$ for synthesis of heavy isotopes of element 118. An analysis of old $\mathrm{Cf}$ sources identified approximate 17 sources that contained high ${ }^{251} \mathrm{C}$ content $(\sim 35 \mathrm{wt} \%)$ with very little remaining ${ }^{252} \mathrm{Cf}$. These sources were retrieved from the storage pool and disassembled. The californium oxide was dissolved in nitric acid and separated and purified for use in the preparation of accelerator target segments for proposed super-heavy element research at Dubna. This research includes a search for ${ }^{295} 118$ and ${ }^{296} 118$, which would be the heaviest nuclei synthesized to date. While this target presents a unique opportunity for the desired experiment, a target highly-enriched $(>95 \%)$ in ${ }^{251} \mathrm{Cf}$ would be preferred, enhancing the production of ${ }^{295} 118$ and ${ }^{296} 118$ while reducing radioactivity compared to the mixed isotope target. This level of enrichment would require an isotope separator capable of handling actinides and the levels of radioactivity associated with this material. An electromagnetic isotope separations unit [53] has been assembled and demonstrated at ORNL for stable isotopes, and several planned upgrades are in progress. A similar unit, with the necessary containment of the ion source and collectors for the separated actinides, would enable expanded research opportunities by providing enriched actinides for super-heavy element synthesis and other research needs, including a possible search for element 120 using ${ }^{251} \mathrm{Cf}+{ }^{50} \mathrm{Ti}$.

Yields of ${ }^{250} \mathrm{Cf}$ and ${ }^{251} \mathrm{Cf}$ from ${ }^{252} \mathrm{Cf}$ production targets follow the same general pattern as that of ${ }^{249} \mathrm{Bk}$, and yields of ${ }^{249} \mathrm{Cf}$ are almost entirely from decay of ${ }^{249} \mathrm{Bk}$. Yields are approximately $0.5 \%$ and $0.1 \%$ of the mass of ${ }^{248} \mathrm{Cm}$ in the target for ${ }^{250} \mathrm{Cf}$ and ${ }^{251} \mathrm{Cf}$ respectively, and reach $90 \%$ of their maximum yield after a single cycle. As with ${ }^{249} \mathrm{Bk}$, yields of these isotopes increase by $40-60 \%$ with the use of a filter providing a $50 \%$ reduction in thermal flux. The yield of ${ }^{252} \mathrm{Cf}$ under such an irradiation scheme is not similarly increased, and is actually reduced by $25 \%$. Therefore production of ${ }^{249-251} \mathrm{Cf}$ using a thermal 
neutron filter would not only increase the yields of these isotopes per target mass, but would also increase their weight percent and reduce the total activity of the separated $\mathrm{Cf}$ fraction.

The production of ${ }^{252} \mathrm{Cf}$ can be further reduced by extending the neutron filter past the $\sim 0.5 \mathrm{eV}$ thermal flux boundary to approximately $10 \mathrm{eV}$. Above $10 \mathrm{eV}$, the capture cross section of ${ }^{251} \mathrm{Cf}$ drops dramatically, with no known epithermal resonances. However ${ }^{252} \mathrm{Cf}$ contains many epithermal resonances, contributing to a capture cross section which is higher than ${ }^{251} \mathrm{Cf}$ in this energy range. As a result, any ${ }^{252} \mathrm{Cf}$ produced from ${ }^{251} \mathrm{Cf}$ is quickly destroyed, minimizing activity in the $\mathrm{Cf}$ fraction. An extended neutron filter could be employed by using a combination of isotopes with overlapping resonances, such as ${ }^{175} \mathrm{Lu}$ and ${ }^{240} \mathrm{Pu}$. Producing tens of $\mathrm{mg}$ of ${ }^{251} \mathrm{Cf}$ by this method would require irradiation of grams of ${ }^{248} \mathrm{Cm}$, either as one large batch or through years of smaller batch irradiations with $\mathrm{Cf}$ recovery and target recycle after each irradiation.

\subsection{Einsteinium and fermium}

Einsteinium ( $\mathrm{Z}=99$ ), if available in sufficient quantities, would provide an excellent path to element 119 (using ${ }^{48} \mathrm{Ca}$ beams), and possibly to element 121 (using ${ }^{50} \mathrm{Ti}$ beams). Currently, einsteinium $\left({ }^{254} \mathrm{Es}\right.$, half-life 276d) can only be produced in microgram quantities, $\sim 3$ orders of magnitude less than typically required for super-heavy element synthesis. Yields of ${ }^{254} \mathrm{Es}$ from ${ }^{252} \mathrm{Cf}$ production are typically in the range of $0.6 \mu \mathrm{g}$ per gram of ${ }^{248} \mathrm{Cm}$ at reactor discharge, along with approximately 30 times as much ${ }^{253} \mathrm{Es}$. The ${ }^{253} \mathrm{Es}\left(\mathrm{T}_{1 / 2}=20.5\right.$ days) is allowed to decay for approximately six months to equal activity with ${ }^{254} \mathrm{Es}$ $\left(\mathrm{T}_{1 / 2}=276\right.$ days), resulting in a usable amount of approximately $0.3 \mu \mathrm{g}$ per gram ${ }^{248} \mathrm{Cm} .{ }^{254 \mathrm{~m}} \mathrm{Es}$ and ${ }^{255} \mathrm{Es}$ are also produced concurrently with ${ }^{254} \mathrm{Es}$, in approximately 10 times greater amounts. However, these isotopes decay with half-lives of $39 \mathrm{~h}$ and $40 \mathrm{~d}$ respectively to ${ }^{254} \mathrm{Fm}$ and ${ }^{255} \mathrm{Fm}$, and are largely depleted prior to separation of the Es fraction.

During a typical ${ }^{252} \mathrm{Cf}$ campaign using available $\mathrm{Am} / \mathrm{Cm}$ feedstock, $\sim 2 \mu \mathrm{g}$ of ${ }^{254} \mathrm{Es}$ are produced with $1 \mu \mathrm{g}$ useable after the required six months decay time. Fermium production under the same conditions is $\sim 2$ picogram ${ }^{257} \mathrm{Fm}$, with about 0.5 picograms useable after the six months decay time.

In the 1980s, a dedicated HFIR campaign was proposed to increase production of ${ }^{254}$ Es for acceleratorbased research on the chemistry and nuclear physics of trans-einsteinium elements. The Large Einsteinium Activation Program (LEAP) [54] would use a tailored irradiation sequence to produce $\sim 40$ micrograms of ${ }^{254} \mathrm{Es}$, about ten times the normal production. This difficult and expensive experiment would require staged, multiphase irradiations in HFIR including:

- Unfiltered irradiation of one gram of ${ }^{252} \mathrm{Cf}$ for one cycle under moderate neutron flux in the beryllium reflector, during which time ${ }^{253} \mathrm{Cf}$ accumulates and decays to ${ }^{253} \mathrm{Es}$, followed by a 20 -day period to allow build-up of ${ }^{253}$ Es.

- Irradiation for five days under maximum flux conditions in the HFIR flux trap followed by a 36-hour decay to build ${ }^{253} \mathrm{Cf}$ to the maximum yield and initiate decay of ${ }^{253} \mathrm{Es}$.

- Irradiation of the target for one cycle using a $\mathrm{Cd}$ thermal neutron filter in the flux trap to transmute ${ }^{253} \mathrm{Es}$ to ${ }^{254} \mathrm{Es}$. The earlier build-up of ${ }^{253} \mathrm{Cf}$ provides a nearly constant resupply of ${ }^{253} \mathrm{Es}$ as it is transmuted during the irradiation, and the Cd filter minimizes the ratio of ${ }^{254} \mathrm{Es}$ depletion to ${ }^{254} \mathrm{Es}$ production due to a large resonance integral but relatively small thermal cross section in ${ }^{253} \mathrm{Es}$.

The resulting quantity of ${ }^{254}$ Es is still several hundred times smaller than the 10-15 mg typically used in super-heavy element experiments. Advances in accelerator and separator technology, such as at the Super-Heavy Element Factory [55] under construction at Dubna, are closing this gap. However, the production of einsteinium in quantities needed for super-heavy element synthesis remains a significant challenge.

The LEAP process as described above would not similarly enhance the production of ${ }^{257} \mathrm{Fm}$ as the irradiation scheme is designed to prevent transmutation past ${ }^{254}$ Es. Alternative irradiation schemes would need to be developed to see similar increases in ${ }^{257} \mathrm{Fm}$ production. 


\section{Target fabrication and performance}

\subsection{Deposition of actinides}

The first actinide target for nuclear studies was ammonium diurante, prepared by precipitation [56]. This target was used by Lise Meitner, Otto Hahn, and Fritz Strassmann in the 1930s to investigate the irradiation of uranium with neutrons. The science and engineering of fabricating actinide targets for the synthesis of super heavy elements (SHE) has progressed significantly since then both in variety and complexity.

There are several important characteristics to consider when selecting techniques for the preparation of actinide targets for production of SHEs. First, given the limited amounts of the isotopically purified actinide materials, it is important that deposition yields are near $100 \%$, and that the deposited material can be recovered [57]. This is especially true for targets made of expensive isotopes such as ${ }^{249} \mathrm{Bk}$ and ${ }^{251} \mathrm{Cf}$ or isotopes in limited supply such as ${ }^{244} \mathrm{Pu}$. Second, it is important to use target support materials that do not have the possibility of interfering with the unambiguous identification of SHE decay events [58]. For this reason, low $\mathrm{Z}$ materials are used for the substrates, such as $\mathrm{Ti}$ and $\mathrm{Al}$, which do not typically transmute to interfering nuclides under irradiation. Third, the use of wheel target assemblies, which rotate to dissipate heat from the high current beams, require the production of multiple segments [58]. Thus the reproducibility of the technique used to produce the segments is very important, since the target performs best if each segment has the same characteristics, such as homogeneity and morphology. Fourth, these actinide targets are often irradiated for long periods of time in order to maximize the use of the limited actinide material, making durability a concern. Fifth, deposition techniques for radioactive target materials usually require use of glove box and/or hot cell environments due to the radiation hazards from weighable amounts of the heavier actinides. Thus the target production technique may need to be designed for limited or remote operations. In summary, the ideal actinide target fabrication technique uses limited material efficiently, uses substrates that do not interfere with detecting the decay events of synthesized SHEs, produces target segments with reproducible characteristics, produces durable targets, and can be remotely performed.

Today, the most commonly employed target fabrication technique is molecular plating [57-62]. A promising technique, still under development, is polymer-assisted deposition [63,64]. These and other actinide target fabrication techniques are briefly discussed below.

Molecular plating. Electrochemical deposition techniques have been widely utilized to fabricate actinide targets for decades [65]. Recent experiments to synthesize new SHEs, such as element 117, are prime examples of this technique [28,45,34]. The electrochemical deposition technique, often called molecular plating, is increasingly being used and investigated for optimization [57-62,65]. This technique involves the deposition of target material from an organic solution using an applied electrical potential. The equipment required for this is simple and can be operated fairly easily within a glove box or hot cell. Deposition yields of greater than $90 \%$ are typically achieved. Molecular plating is often used to deposit rare actinide materials for this reason. Isopropanol or isobutanol are used as the solvent with $\mathrm{Ti}, \mathrm{Al}$, or $\mathrm{C}$ as the substrate material. Targets prepared by this technique can have thicknesses ranging from about 10 to $1000 \mu \mathrm{g} / \mathrm{cm}^{2}$. Thicker targets have been prepared by molecular plating but require multiple depositions [62]. Improvements to uniformity and adherence to the substrate have been accomplished by using high current densities $\left(\sim 2\right.$ to $\left.6 \mathrm{~mA} / \mathrm{cm}^{2}\right)$. Both adhesion to the substrate and non-homogeneity are issues for molecular plating of thicker targets. One method used to address the adhesion problem has been to sandwich two targets together to prevent deposited material from falling out.

Polymer-assisted deposition. Recently, polymer assisted-deposition has been investigated as a new method to prepare actinide targets $[63,64]$. In this method, a solution containing metal ions chelated to a multi-dentate polymer is prepared. The solution is then spin coated and annealed onto a substrate material, leaving behind a thin metal oxide film. Studies by M. A. Garcia et al. [63,64] have used 
polyethylenimine as the polymer to apply this technique to lanthanides as surrogates for actinides. In contrast to molecular plating, polymer assisted deposition can be used to produce homogenous and uniform films over a wider range of thicknesses, Although polymer assisted deposition does not have yields greater than about $30 \%$ on the first run, the process can be repeated to reach an overall deposition yield close to $100 \%$.

While molecular plating is the dominant target preparation technique today, and polymer-assisted deposition shows great promise, there are many other techniques that have been and can be used to prepare actinide targets [65-68].

Sputtering. Vapor deposition (VD) techniques, such as ion beam sputtering, have been used previously $[65,66]$. In these techniques the target materials are either physically or chemically put into the gas phase and deposited onto the substrate. VD techniques can have inefficient yields that inevitably lead to unrecoverable materials, so this technique is not preferred for the preparation of actinide targets. Additionally, contamination is a significant issue with VD techniques, and complicated equipment is required (i.e. high-vacuum, ion sources, etc.). A major benefit of this technique, however, is the ability to prepare very thin, homogeneous films.

Painting. Another target fabrication method is painting, in which a solution containing the target material is painted onto the substrate using a brush [67]. Understandably, it is difficult to paint a very homogenous and uniform target. However, a benefit of this method is that it can be used to fabricate large area targets. Typical thicknesses for this technique are several hundred $\mu \mathrm{g} / \mathrm{cm}^{2}$. Sedimentation has also been used but is restricted to prepare only extremely thick $\left(\sim 1 \mathrm{~g} / \mathrm{cm}^{2}\right)$ targets.

Electrophoresis. Electrophoresis is a technique particularly suitable for the quick preparation of very thick $\left(\sim 15 \mathrm{mg} / \mathrm{cm}^{2}\right)$ targets [65]. Drawbacks of this technique include the required preparation of a colloidal suspension.

Electrospray. Electrospraying is a technique capable of high deposition yields as well as for preparing homogenous and uniform films. However, the impurities from the solvents and the fact that only compounds can be deposited are major drawbacks as well as significant loss of material during spraying.

Intermetallics. Intermetallic targets can provide improved electrical conductance, heat conductance, and mechanical stability, especially for thick, stationary targets preferred for chemical studies of superheavy elements [68]. The target material is electroplated on a thin metal foil and reduced at high temperature in a flow of pure hydrogen, forming an intermetallic compound with the metal foil. Such targets have been prepared for ${ }^{243} \mathrm{Am}$ on $\mathrm{Pd}$ and successfully tested [68] using intense ${ }^{48} \mathrm{Ca}$ beams at Dubna. Intermetallic targets appear to be more stable than other targets, but potential issues related to formation of pin holes during the reduction process (especially for thick targets) and increased spreading of the target material into the support film need to be considered. The spreading could lead to inefficient use of the target material, since the optimal energy range for a typical fusion excitation function may be smaller than the energy loss in the intermetallic region.

Other potential approaches for the production of high intensity actinide targets include sintering of the target material, preparation of targets on superhydrophic surfaces, and the use of graphene substrates. Sintering [69] of actinide powders could result in robust, high melting temperature actinide oxide coatings on substrate materials. Issues of surface roughness and adhesion to the substrate will need to be resolved. Superhydrophobic surfaces facilitate the homogeneous distribution of target material in dissolved form, from which a uniform film can be evaporated [70] and perhaps sintered. Graphene [71] is a potentially interesting support material due to its very high strength allowing very thin substrates. This can reduce target temperature since, for current actinide target systems, the ${ }^{48} \mathrm{Ca}$ beam deposits most of its energy in much thicker titanium backing [72]. Actinide deposition techniques appropriate for carbon films should work for graphene, and large sheets of graphene are becoming available [73]. 


\subsection{Performance}

Actinide targets fabricated by molecular plating, painting, and more recently intermetallic formation have proven to be very robust. In typical experiments, actinide losses over the course of heavy ion irradiations to fluences of $1 \times 10^{19}$ ions $/ \mathrm{cm}^{2}$ and higher are typically less than $5 \%$ [32]. Some of these losses are due to sputtering and to multinucleon transfer reactions that have higher cross sections compared to fusion reactions. Temperature is another key issue, and intense irradiations often require rotating targets to spread the heat loads. Current rotating target technology [74] at GSI typically limits the surface temperature of the actinide films to less than $100{ }^{\circ} \mathrm{C}$ for beam currents of $\sim 1$ part. $\mu \mathrm{A}$. Tests at Dubna [29] have demonstrated that ${ }^{48} \mathrm{Ca}$ beam currents up to 5-6 part. $\mu \mathrm{A}$ and doses up to $5 \times 10^{19}$ ions can be tolerated spread over target areas of $32 \mathrm{~cm}^{2}$.

The low production cross sections for heavier super-heavy elements, including the use of new ion beams such as ${ }^{50} \mathrm{Ti}$ and ${ }^{54} \mathrm{Cr}$, will require increased ion beam currents and fluences for next generation super-heavy element experiments. Anticipated beam currents of tens of $\mu \mathrm{A}$ will deposit hundreds of watts of power in the target and backing, exceeding the performance limits of many existing target systems. While the use of larger target wheels can help reduce target heating, it is not possible to continue spreading the beam across increasing target areas for the heaviest actinides, including ${ }^{249} \mathrm{Bk}$ and ${ }^{249-251} \mathrm{Cf}$, due to the limited availability of these isotopes. Strategies to accommodate higher ion beam currents include enabling the utilization of larger target wheels by exploiting techniques discussed in Section 3 to increase the availability of rare actinides, research on new material forms to accommodate higher target temperatures, and enhanced cooling [75] to reduce target temperatures during irradiation. Target mechanical integrity, morphological stability, emissivity, and temperature variation must also be addressed [72]. The practical limits of these strategies need to be explored.

\section{Summary}

Over the past 70 years, the Periodic Table has been expanded by more than $25 \%$, as 26 new, artificially created elements have been observed. Some of these new elements, such as plutonium and californium, have enabled important technological advances, while others have contributed to significant scientific progress. All have been made possible by continued advances in target materials and technology, with actinide targets contributing to 20 of these discoveries, including 9 of 15 super-heavy elements.

In the past decade, progress in the development of heavy actinide targets has contributed to the discovery of six new elements, elements 113-118, and more than 50 new isotopes. These discoveries have validated theoretical predictions of nuclear structure evolution and have probed the outer boundary of the island of enhanced nuclear stability. They depend on a robust infrastructure of actinide production, chemical processing, and heavy ion accelerator facilities. Continued scientific progress will require further advances in actinide production, processing, and target technology to enable experiments essential for exploring the limits of nuclear stability, heavy element chemistry, and the Periodic Table.

\section{Acknowledgments}

This research was supported by the U.S. Department of Energy (DOE) Office of Nuclear Physics, Isotope Development and Production for Research and Applications Program, under contract DE-AC0500OR22725 with UT-Battelle, LLC. We are grateful to the staffs of the ORNL Radiochemical Engineering Development Center and High Flux Isotope Reactor, a DOE Office of Science User Facility, for their support in the production and chemical separation of the actinide materials. We also thank our many collaborators at the Flerov Laboratory of Nuclear Reactions (JINR, Dubna, Russia), GSI (Darmstadt, Germany), University of Mainz (Mainz, Germany), Lawrence Livermore National 
Laboratory, Vanderbilt University, and the University of Tennessee-Knoxville, without whom this research would not have been possible.

\section{References}

[1] G. T. Seaborg, Chem. Eng. News 23, 2190 (1945)

[2] G. T. Seaborg, Science 104, 379 (1946)

[3] N. N. Greenwood and Alan Earnshaw, Chemistry of the Elements ( $2^{\text {nd }}$ edition), Butterworth Heinemann, ISBN 0080379419 (1997)

[4] G. T. Seaborg, Nucl. Sci. and Eng. 9, 475 (1961)

[5] A. Ghiorso et al., Phys Rev 99, 1048 (1955)

[6] A. Ghiorso, Phys. Rev. 98, 1518 (1955)

[7] E. D. Donets, V. A. Shchegolov, and V. A. Ermakov, Sov. At. Energy 20, 257 (1966)

[8] B. A. Zager et al., Sov. At. Energy 20, 264 (1966)

[9] A. Ghiorso, T. Sikkeland, A. E. Larsh, and R. M. Latimer, Phys. Rev. Lett. 6, 473 (1961)

[10] E. Donets et al., Sov. At. Energy 19, 995 (1965)

[11] G. N. Flerov et al., Phys. Letters 13, 73 (1964)

[12] I. Zavara, Y. T. Chuburkov, R. Caletka, and M. R. Shalaevsky, Radiokhimiya 11, 163 (1969)

[13] A. Ghiorso et al., Phys. Rev. Lett. 22, 1317 (1969)

[14] A. Ghiorso et al., Phys. Rev. Lett. 24, 1498 (1970)

[15] G. N. Flerov et al., Sov. At. Energy 29, 967 (1970)

[16] A. Ghiorso, Phys. Rev. Lett. 33, 1490 (1974),

[17] Yu. Ts. Oganessian, JETP Lett. 20, 580 (1974)

[18] G. Muenzenberg et al., Zeitschrift fuer Physik A 300, 107 (1981)

[19] G. Muenzenberg et al., Zeitschrift fuer Physik A 317, 235 (1984)

[20] G. Muenzenberg et al., Zeitschrift fuer Physik A 309, 89 (1982)

[21] S. Hofmann et al., Zeitschrift fuer Physik A 350, 277 (1995)

[22] S. Hofmann et al., Zeitschrift fuer Physik A 350, 281 (1995)

[23] S. Hofmann et al., Eur. Phys. J. A 147, (2002)

[24] Yu. Ts. Oganessian et al., Phys Rev C 62, 041604 (2000)

[25] Yu. Ts. Oganessian et al., Phys. Rev. C 69, 021601 ((2004)

[26] Yu. Ts. Oganessian et al., Phys. Rev. C 69, 054607 (2004)

[27] Yu. Ts. Oganessian et al., Phys. Rev. C 74, 044602 (2006)

[28] Yu. Ts. Oganessian et al., Phys. Rev. Lett. 104, 142502 (2010)

[29] Yu. Ts. Oganessian et al., J. Phys. G 34, R165 (2007)

[30] L. Stavsetra et al., Phys. Rev. Lett. 104, 132502 (2009)

[31] Ch. E. Duellmann et al., Phys. Rev. Lett. 103, 252701 (2009)

[32] D. Rudolph et al., Phys. Rev. Lett. 111, 112502 (2013)

[33] S. Hofmann et al., Eur. Phys. J. A 48, 62 (2012)

[34] J. Khuyagbaatar et al., Phys. Rev. Lett. 112, 172501 (2014)

[35] K. Morita et al., J. Phys. Soc. Japan 81, 2593 (2004)

[36] M. Thoennessen, Atomic Data and Nuclear Tables 99, 312 (2012)

[37] J. B. Roberto et al., in Fission and Properties of Neutron-Rich Nuclei, J. H. Hamilton and A. V. Ramayya, eds., World Scientific, ISBN 978-981-4525-42-8, pp. 287-294 (2014)

[38] I. W. Osborne- Lee and C. W. Alexander, ORNL/TM-12760 (1995)

[39] R. G. Haire in The Chemistry of the Actinide and Transactinide Elements, 4th ed., L. R. Morss, N. M. Edelstein, and J. Fuger, eds., Springer (Netherlands), pp. 1265-1395 (2011)

[40] J. E. Bigelow, E. D. Collins, and L.J. King in Actinide Separations, J. D. Navratil and W. W. Schulz, eds., ACS Symp. Ser. 117, American Chemical Society, Washington, DC, pp. 147-55 (1980) 
[41] E. D. Collins, D. E. Benker, F. R. Chattin, P. B. Orr, and R. G. Ross in Transplutonium Elements-Production and Recovery, J. D. Navratil and W. W. Schulz, eds., ACS Symp. Ser. 161, American Chemical Society, Washington, DC, pp. 147-60 (1981)

[42] E. K. Hulet, R. G. Gutmacher, and M. S. Coops, J. Inorg. Nucl. Chem. 17, 350-60 (1961)

[43] D. E. Benker, F. R. Chattin, E. D. Collins, J. B. Knauer, P. B. Orr, R. G. Ross and J. T. Wiggins, in Transplutonium Elements-Production and Recovery, J. D. Navratil and W. W. Schulz, eds., ACS Symp. Ser. 161, American Chemical Society, Washington, DC, pp. 161-72 (1981)

[44] G. R. Choppin, B. G. Harvey and S. G. Thompson, J. Inorg. Nucl. Chem., 2, 66-68 (1956)

[45] Yu. Ts. Oganessian et al., Phys. Rev. Lett. 109, 162501 (2012)

[46] Yu. Ts. Oganessian et al., Phys. Rev. C 87, 054621 (2013)

[47] S. Dmitriev, presented at Zakopane Conference on Physics, Zakopane, Poland (2010)

[48] Ch. E. Duellmann, in Fission and Properties of Neutron-Rich Nuclei, J. H. Hamilton and A. V. Ramayya, eds., World Scientific, ISBN 978-981-4525-42-8, pp. 271-277 (2014)

[49] J. Chen, I. Ahmad, J. P. Greene, and F. G. Kondev, Phys. Rev. C 90, 044302 (2014)

[50] S. F. Mughabghab, Atlas of Neutron Resonances, $5^{\text {th }}$ ed., Elsevier BV (2006)

[41] M. B. Chadwick et al., ENDF/B-VII.1 Nuclear Data for Science and Technology: Cross Sections, Covariances, Fission Product Yields and Decay Data, Nuclear Data Sheets, 112, 2887 (2011)

[52] S. Hogle, C. W. Alexander, J. D. Burns, J. G. Ezold, “Experimental Validation for Optimization of Transcurium Isotope Production," presented at $8^{\text {th }}$ International Conference on Isotopes, Chicago, IL (2014)

[53] B. J. Egle, K. J. Hart, and W. S. Aaron, J. Radioanal. Nucl. Chem. 299, 995 (2014)

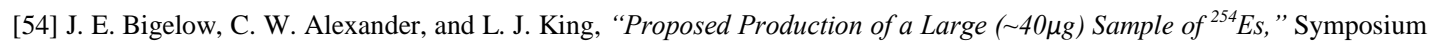
on Heavy Element Research, AIChE Summer National Meeting, Seattle, Washington (1985)

[55] http://flerovlab.jinr.ru/flnr/she_factory.html

[56] G. Hermann, Nucl. Instr. Meth. A 282 (1989)

[57] J. Runke, et al., J. Radioanal. Nucl. Chem. 299, 1081 (2014)

[58] K. Eberhardt et al., Nucl. Instr. Meth. in Phys. Res. A 590, 134 (2008)

[59] K. Eberhardt et al., Nucl. Instr. Meth. in Phys. Res. A 521, 208 (2004)

[60] A. Vascon et al., Nucl. Instr. Meth. in Phys. Res. A 655, 72 (2011)

[61] P. A. Ellison, et al., Phys. Rev. Lett. 105, 182701 (2010)

[62] Q. Zhi et al., Applied Radiation and Isotopes 54, 741 (2001)

[63] M. A. Garcia et al., Thin Solid Films 516, 6261 (2008)

[64] M. A. Garcia et al., Nucl. Instr. Meth. in Phys. Res. A 613, 396 (2010)

[65] K. M. Glover, Nucl. Instr. Meth. in Phys. Res. A 236, 435 (1985)

[66] J. Kwinta, Nucl. Instr. Meth. 167, 65 (1979)

[67] L. V. Drapchinsky et al., Nucl. Instr. Meth. in Phys. Res. A 438, 116 (1999)

[68] I. Usoltsev et al., Nucl. Instr. Meth. in Phys. Res. B 318, 297 (2014)

[69] R. W. Cahn and P. Haasen, Physical Metallurgy (4 ${ }^{\text {th }}$ ed.), ISBN 978-0-444-89875-3 (1996), pp 2399-2500

[70] D. Renisch et. al., Nucl. Instr. Meth. in Phys. Res. A 676, 84 (2012)

[71] A. K. Geim, Science 324, 1530 (2009)

[72] Ch. Stodel et al., Nucl. Instr. Meth. in Phys. Res. A 613, 480 (2010)

[73] I. Vlassiouk et al., Carbon 54, 58 (2013)

[74] E. Jager et al., J. Radioanal. Nucl. Chem. 299, 1073 (2014)

[75] S. Antalic et al., Nucl. Instr. Meth. in Phys. Res. A 530, 185 (2004) 